\title{
Shock-induced reaction in a flake nickel + spherical aluminum powder mixture
}

D. Eakins and N.N. Thadhani

Reprinted with permission from D. Eakins, Journal of Applied Physics, 100, 113521 (2006). Copyright 2006, American Institute of Physics. This article may be downloaded for personal use only. Any other use requires prior permission of the author and the American Institute of Physics. 


\title{
Shock-induced reaction in a flake nickel + spherical aluminum powder mixture
}

\author{
D. Eakins and N. N. Thadhani ${ }^{\text {a) }}$ \\ School of Materials Science and Engineering, Georgia Institute of Technology, 771 Ferst Drive, \\ Love Manufacturing Building, Atlanta, Georgia 30332
}

(Received 24 July 2006; accepted 14 September 2006; published online 11 December 2006)

\begin{abstract}
Mixtures of flake-shaped nickel and spherical aluminum powders have been subjected to shock-loading up to $6 \mathrm{GPa}$ to investigate the occurrence of shock-induced chemical reactions. The high-pressure Hugoniot state is determined through time-resolved measurements of the input stress and shock transit time through the specimen. An increase in shock velocity is observed above stress levels of $3.5 \mathrm{GPa}$, suggesting the initiation of an ultrafast shock-induced chemical reaction and formation of $\mathrm{Ni}-\mathrm{Al}$ compound(s) occurring in the time scale of the high-pressure shock state. (C) 2006 American Institute of Physics. [DOI: 10.1063/1.2396797]
\end{abstract}

\section{INTRODUCTION}

The initiation of chemical reactions within or shortly behind the shock front in intermetallic-forming mixtures has been the subject of investigations employing instrumented experiments for nearly 20 years. ${ }^{1-14}$ These aptly named "shock-induced" reactions represent a unique class of chemical behavior, whereby intrinsic and extrinsic component properties play a significant role in controlling reaction initiation. Intrinsic properties are material-inherent properties such as density, flow strength, and elastic properties, and are determined by component selection. Extrinsic properties, on the other hand, are descriptions of particle condition and powder configuration, which serve to modify the mechanical/chemical response. ${ }^{15}$ Examples of extrinsic properties include particle size and morphology, powder mixture packing density, component distribution, and particle pretreatment.

It is proposed that the initiation of shock-induced chemical reactions is linked to mechanochemical phenomena, such as the degree of short time-scale mixing. Prior work has demonstrated that the highest degree of mixing can be achieved through matched intrinsic properties, i.e., comparable yield strength and densities. Systems comprised of highly mechanically differing constituents exhibit heterogeneous deformation or fragmentation, and consequently limited mixing and lack of shock-induced reaction. ${ }^{10,12,16,17}$ The nickel-aluminum powder system represents a mixture of hard and soft phases, where the static yield strength and densities differ by more than $200 \%$. With such intrinsically influencing variables, and in the case of spherically-shaped micronscale particles at 60\% TMD (theoretical maximum density), no evidence of shock-initiated reaction has been observed in our prior work, albeit at shock input stresses up to $6 \mathrm{GPa}^{18,19}$ However, it is believed that enhanced interparticle mixing and reaction initiation can be facilitated through adjustment of extrinsic properties. The influence of extrinsic properties, and in particular particle morphology, on deformation and mixing in the nickel-aluminum system has thus far only been investigated through recovery experiments. ${ }^{16,17}$ Post-mortem microstructural analysis of recovered samples in these ex- periments has revealed compound formation in flake-shaped $\mathrm{Ni}+3 \mathrm{Al}$ powders at higher packing densities than in spherical powder mixtures subjected to similar peak shock pressures, indicating a lower shock energy required for compound formation with the flaky morphology powders. Results of recovery experiments however, do not provide any evidence of reaction occurrence and compound formation during or following the high pressure shock state.

The work presented in this paper describes the first timeresolved instrumented Hugoniot measurements performed on the nickel-aluminum system incorporating flake-shaped $\mathrm{Ni}$ particles to investigate the effect of component extrinsic properties on the shock-initiation of chemical reactions.

\section{EXPERIMENTAL PROCEDURE}

Time-resolved Hugoniot measurements were performed using the parallel-plate impact experiment configuration (Fig. 1), in which the impact of a $\mathrm{Cu}$ or $\mathrm{W}$ flyer against a $\mathrm{Cu}$ driver plate (nominally $57.2 \mathrm{~mm}$ diameter, $5.6 \mathrm{~mm}$ thick) produces a well-defined shock pulse that propagates unattenuated through a thin $(\sim 2.5 \mathrm{~mm}$ thick) powder layer sandwiched between two polyvinylidene difluoride (PVDF) stress gauges. Table I lists the experimental conditions and dimensions of the flyer-sample configuration. The complete details of experimental configuration and the measurement system are presented in earlier publications. ${ }^{11,18}$ The specimens consisted of -325 mesh Ni flakes ( 0.3 $\mu$ m thick), mixed with -325 mesh $\mathrm{Al}$ rounded particles at a ratio of 1:1 by volume. The initial packing density was $45 \pm 2 \%$ TMD. Micrographs showing the starting and pressed powder configurations are shown in Fig. 2. The Hugoniot state was determined through PVDF gauge measurements of the input stress $P$ and traveltime through the powder thickness to obtain a measure of the shock speed $U_{s}$. Inference of the reaction product Hugoniot was made based upon the inert Hugoniot calculated through mixture theory, ${ }^{20}$ and the Ballotechnic model ${ }^{21}$ for the limits of complete reaction. The Ballotechnic model performs a constant pressure adjustment of the mixture Hugoniot in proportion to the heat of reaction of the product phase. The heats 
(a)
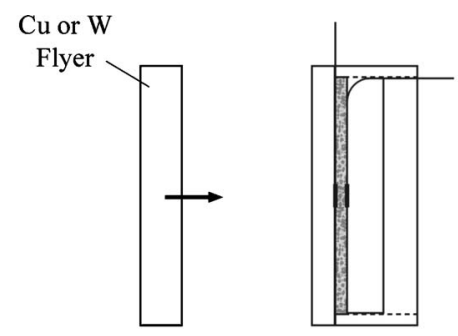

Input

(b)

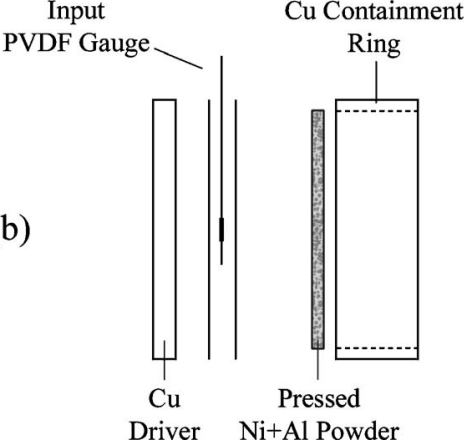

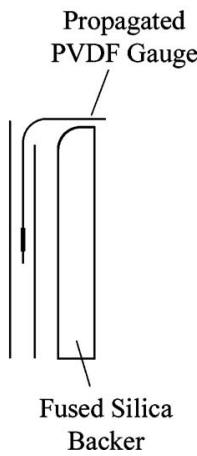

Backer

FIG. 1. Illustration of the parallel-plate impact experiment, showing (a) flyer-target assembly and (b) exploded view of the sample assembly. The input and propagated PVDF stress gauges are in direct contact with the powder layer of nominal thicknesses listed in Table I for each experiment.

of reaction used in the Ballotechnic model for the phases considered $\mathrm{NiAl}, \mathrm{NiAl}_{3}$, and $\mathrm{Ni}_{3} \mathrm{Al}$, are respectively 1.38 , 1.08 , and $0.753 \mathrm{~kJ} / \mathrm{g}$.

\section{RESULTS AND DISCUSSION}

Figure 3 shows the input and propagated stress profiles obtained by converting the current traces recorded by PVDF stress gauges, using the Sandia software PlotData. ${ }^{22}$ The multiple wave reflections within the gauge package introduce reverberations in the input traces. It should be noted that as documented in previous works, ${ }^{10-12}$ the propagated waves are quite dispersed with shock rise times of 30-80 ns. However, the flyer-driver-sample geometry (aspect ratio of 20:1) ensured that the shock wave propagating through the powder sample layer thickness remains unattenuated and is unaffected by two-dimensional radial effects. Selection of the input stress followed the method described previously by $\mathrm{Xu}$ and Thadhani. ${ }^{11}$ This method accounts for the reverberations

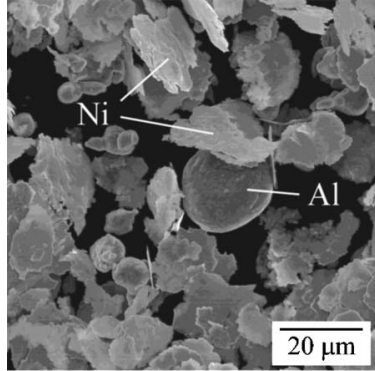

(a) starting

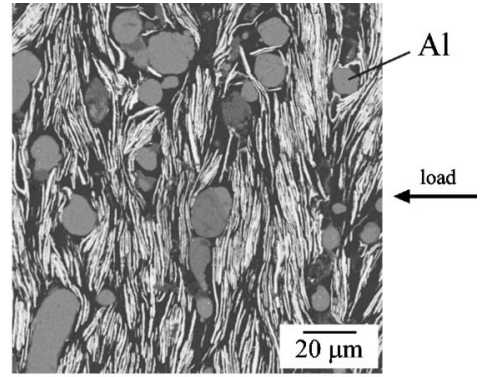

(b) as-pressed
FIG. 2. Scanning electron micrographs of the flake $\mathrm{Ni}$ and spherical $\mathrm{Al}$ mixture in (a) starting, unmixed condition, and (b) after pressing to the initial density of $45 \%$ TMD. Note that the flakes have aligned perpendicular to the pressing direction.

caused by multiple reflections within the gauge package, and requires that the input stress be taken beyond this reverberation interval. The end of the reverberation interval is identified in the current trace by the time (typically 90-150 ns) corresponding to a $90 \%$ decrease in the maximum reverberation height. Shock velocities were measured based on the travel time between the two gauges, less the time through the gauge package insulation. Table I lists the measured impact velocity, input stress, and shock velocity obtained from the stress gauge records, for each experiment. The measured shock velocity and the input stress data points are also plotted in Fig. 4, along with the calculated Hugoniot of the inert mixture of $\mathrm{Ni}+\mathrm{Al}$ and that of reaction products corresponding to each of the possible $\mathrm{Ni}_{3} \mathrm{Al}$, $\mathrm{NiAl}$, and $\mathrm{NiAl}_{3}$ compounds. Note that the data points plotted represent lower limits, i.e., conservative estimates of the shock velocity.

The data points measured at low stress (up to $3.5 \mathrm{GPa}$ ), remain close to the calculated Hugoniot of the inert mixture. Above $3.5 \mathrm{GPa}$, increases in shock velocity revealed by deviation from the inert trend are observed. As shown in the inset in Fig. 4, the data lie entirely within the limits of complete reaction product formation at input stresses of 5.3 and $5.4 \mathrm{GPa}$. These results indicating an increase in shock velocity suggest the occurrence of inert-deviant behavior, and in particular, the "shock-induced" formation of intermetallic phases in the vicinity of the shock front. Changes in shock velocity associated with phase transformations such as

TABLE I. Parallel-plate impact experiment data.

\begin{tabular}{ccccccc}
\hline \hline Shot No. & Flyer type $^{\mathrm{ab}}$ (thickness, mm) & $\begin{array}{c}\text { Sample thickness } \\
(\% \mathrm{TMD})\end{array}$ & $\begin{array}{c}\text { Impact velocity } \\
(\mathrm{m} / \mathrm{s})\end{array}$ & $\begin{array}{c}\text { Input stress } \\
(\mathrm{GPa})\end{array}$ & $\begin{array}{c}\text { Shock velocity }^{\mathrm{c}} \\
(\mathrm{mm} / \mu \mathrm{s})\end{array}$ \\
\hline 0532 & $\mathrm{Cu}$ & $(9.2)$ & $2.41(45.7)$ & $1004 \pm 4$ & 3.5 & $1.566 \pm 0.005$ \\
0539 & $\mathrm{Cu}$ & $(8.7)$ & $2.42(44.6)$ & $669 \pm 3$ & 1.9 & $1.190 \pm 0.005$ \\
0540 & $\mathrm{~W}$ & $(5.1)$ & $2.50(45.1)$ & $951 \pm 5$ & 5.4 & $2.136 \pm 0.12$ \\
0606 & $\mathrm{~W}$ & $(5.1)$ & $2.40(47.3)$ & $912 \pm 4$ & 4.9 & $1.892 \pm 0.038$ \\
0607 & $\mathrm{Cu}$ & $(9.1)$ & $2.47(44.6)$ & $430 \pm 3$ & 0.7 & $0.753 \pm 0.007$ \\
0612 & $\mathrm{~W}$ & $(5.2)$ & $2.27(47.6)$ & $1012 \pm 8$ & 5.5 & $2.146 \pm 0.085$ \\
\hline \hline
\end{tabular}

${ }^{\mathrm{a}} \mathrm{Cu}$, nominally $57.2 \mathrm{~mm}$ diameter, $9 \mathrm{~mm}$ thick.

${ }^{\mathrm{b}} \mathrm{W}$, nominally $50.8 \mathrm{~mm}$ diameter, $5.1 \mathrm{~mm}$ thick.

${ }^{\mathrm{c}}$ Error range in shock velocity due to measurements of sample thickness and arrival time. 
(a)
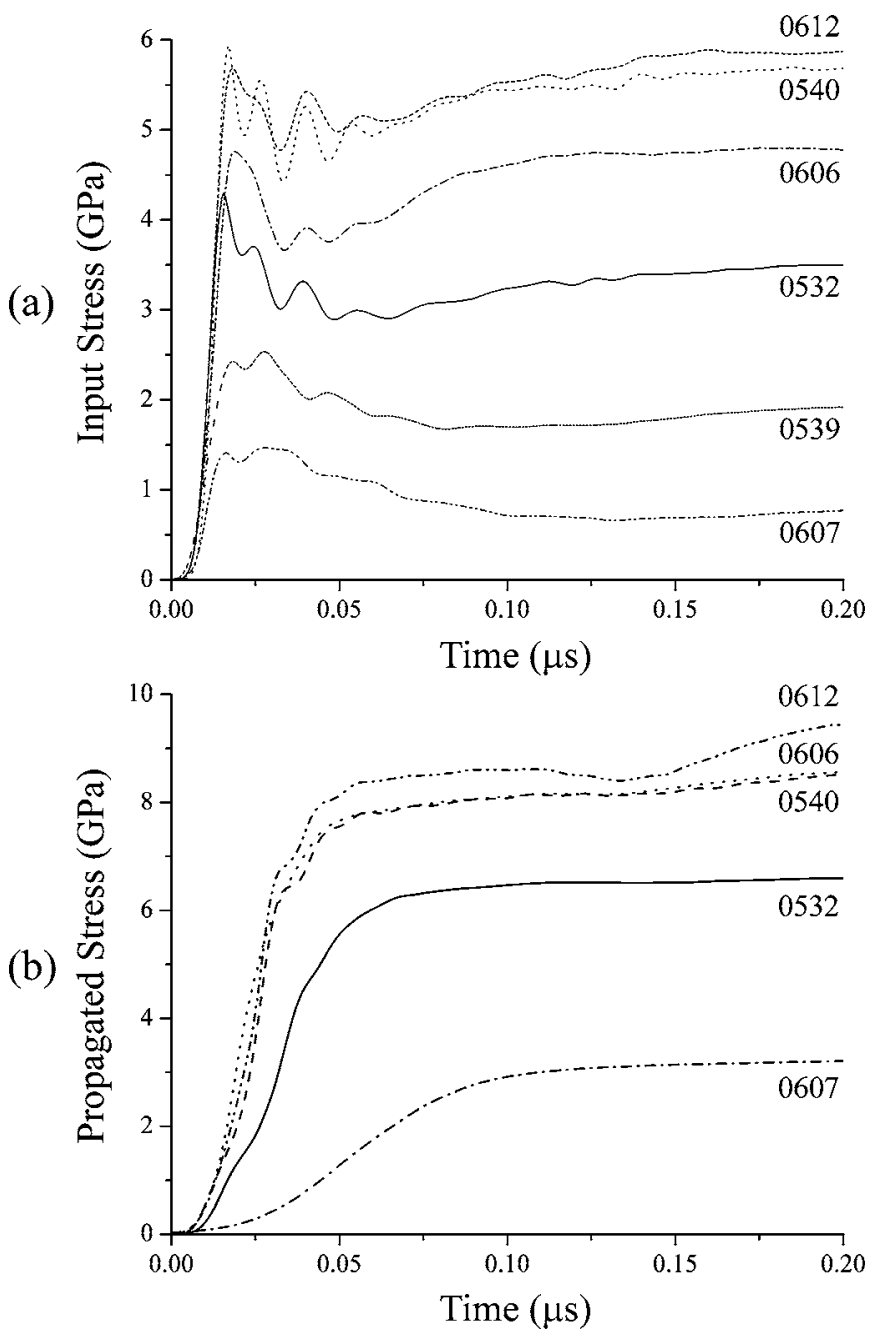

FIG. 3. Stress profiles obtained from PVDF gauges mounted on the front and rear specimen surface. The input gauge records (a) exhibit low rise times $(<10 \mathrm{~ns})$, and reverberations arising from the many reflections through the low impedance gauge package sandwiched between the copper driver and the powder mixture. These reverberations are absent from the propagated gauge stress profiles (b), due to the much longer rise times $(30-80 \mathrm{~ns})$ caused by dispersion of the shock front as it traverses through the thickness of the powder layer.

melting, have been shown to produce a decrease in shock velocity, as reported in the $\mathrm{Mo}-\mathrm{Si}$ and $\mathrm{Nb}-\mathrm{Si}$ systems. ${ }^{12,23}$ Inference of shock-induced reaction initiation based on shock velocity increases associated with compound formation in binary powder mixtures, has also been reported in our prior work, ${ }^{10,11}$ as well as more recently by Gur'ev and co-workers. ${ }^{13,14}$

The degree of reaction and product phases formed due to shock-induced reactions cannot be determined directly through the instrumented experiments performed in this work. Based upon the apparent increase in shock velocity however, an initial attempt was made to construct a reactioncomposition surface, corresponding to the concentrations and extent of possible product phases. By assuming that the observed shock velocity is a combination of the shock velocities through inert and reacted phases (see Appendix), the overall degree of reaction $X^{r x n}$ can be deduced from the following equation:

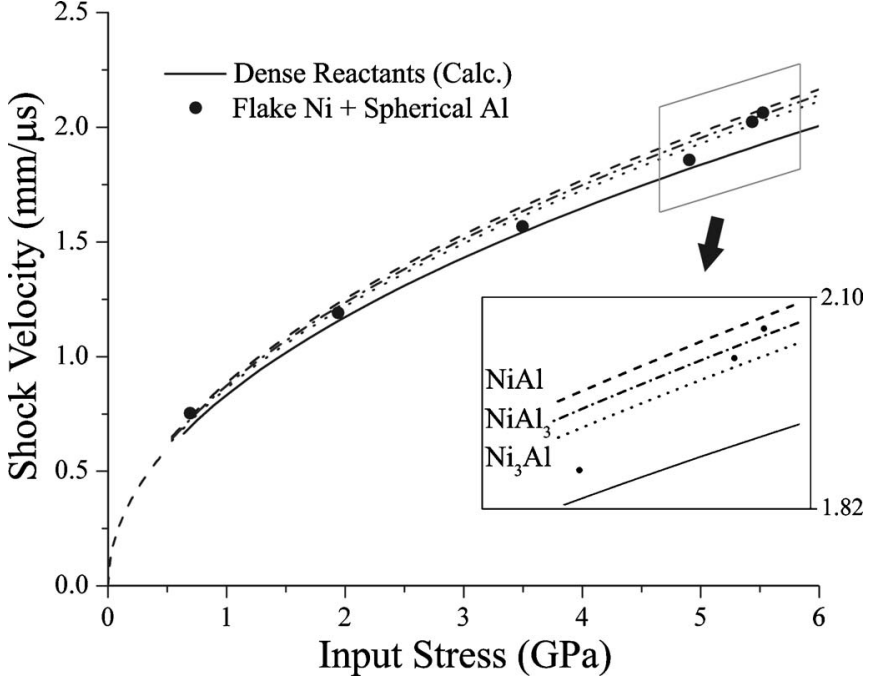

FIG. 4. Plot of shock velocity and input stress for the flake mixtures up to 6 $\mathrm{GPa}$. The experimental data (closed circles) follow the calculated inert response (solid line) up to $3.5 \mathrm{GPa}$. The data at higher stresses (inset) indicate a deviation toward the calculated reaction product curves (dashed lines) suggesting the occurrence of shock-induced reaction.

$$
\begin{aligned}
& X^{r x n}=\frac{U_{s}^{r x n} \cdot U_{s}^{0}}{U_{s}^{\mathrm{obs}}\left(U_{s}^{0}-U_{s}^{r x n}\right)}-\frac{U_{s}^{r x n}}{\left(U_{s}^{0}-U_{s}^{r x n}\right)}, \\
& X^{r x n}=\frac{U_{s}^{r x n} \cdot U_{s}^{o}}{U_{s}^{\mathrm{obs}}\left(U_{s}^{o}-U_{s}^{r x n}\right)}-\frac{U_{s}^{r x n}}{\left(U_{s}^{o}-U_{s}^{r x n}\right)},
\end{aligned}
$$

where $U_{s}^{\text {obs }}$ is the shock velocity observed experimentally, $U_{s}^{o}$ is the shock velocity of an inert mixture at the observed pressure, and $U_{s}^{r x n}$ the combined shock velocity from the reaction product phases $\beta, \delta$, and $\gamma$, is defined by

$$
U_{s}^{r x n}=\frac{U_{s}^{\beta} \cdot U_{s}^{\delta} \cdot U_{s}^{\gamma}}{X^{\beta} U_{s}^{\delta} \cdot U_{s}^{\gamma}+X^{\delta} U_{s}^{\beta} \cdot U_{s}^{\gamma}+X^{\gamma} U_{s}^{\beta} \cdot U_{s}^{\delta}},
$$

where $X^{\beta}, X^{\delta}$, and $X^{\gamma}$ are the relative volume fractions of the phases present in the reacted product $X^{r x n}$. Figure 5 shows such a reaction-composition diagram, derived from the data points above $3.5 \mathrm{GPa}$. The horizontal surface is a ternary composition space for the $\mathrm{NiAl}, \mathrm{Ni}_{3} \mathrm{Al}$, and $\mathrm{NiAl}_{3}$ phases, whereas the vertical axis represents the reaction extent (essentially a composition balance between unreacted and reacted material).

Considering the deviation observed from experiment 0606 at $4.9 \mathrm{GPa}$, any combination of the phases may have existed as illustrated in Fig. 5, though no more than $43 \%$ of the material could have undergone reaction. The experiments conducted at $5.4(0540)$ and $5.5 \mathrm{GPa}(0612)$ indicate a greater extent of reaction, however as revealed by the diminishing surfaces, it now seems unlikely that only $\mathrm{NiAl}_{3}$ or $\mathrm{Ni}_{3} \mathrm{Al}$ are present. Furthermore, if the reaction mechanism(s) is continuous for each of the three reacted samples, then the product phases formed are predominantly $\mathrm{NiAl}$ in composition. Mesoscale discrete particle simulations of shock compression of powder mixtures, is currently being performed in our group to determine the extent of reaction and reaction products, and further refine this analysis. 


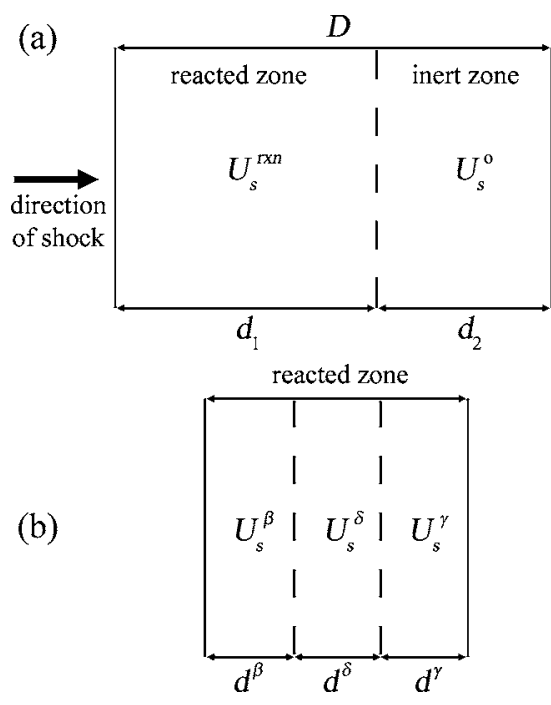

FIG. 6. Schematic used to derive the (a) extent of reaction, and (b) concentration of reaction products phases. The net influence of reaction on shock velocity is approximated by considering parallel layers of inert and reacted material.

\section{CONCLUSIONS}

Parallel-plate impact experiments have been performed on micron-scale flake-shaped nickel and spherical aluminum powder mixtures up to $6 \mathrm{GPa}$ to investigate the role of particle morphology as an extrinsic property on shock-induced chemical reactions based on time-resolved input stress and shock velocity measurements. Deviations between the calculated Hugoniot of inert reactants and data reduced from PVDF gauge records were observed above $3.5 \mathrm{GPa}$, indicating shock velocity increase associated with shock-induced chemical reaction. Based on the limits of complete reaction calculated using the Ballotechnic model, and a preliminary reaction-composition analysis, the results suggest shockinduced formation of the intermetallic compound with the predominant phase being equiatomic NiAl. With the appar-

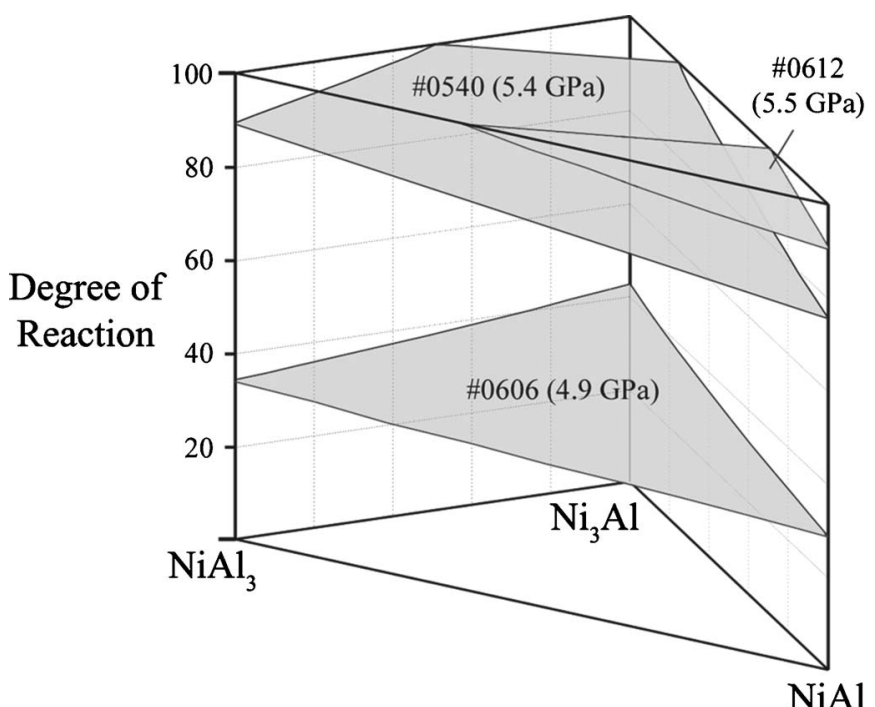

FIG. 5. Ternary reaction-composition diagram showing the possible concentrations and reaction extent based upon the deviations in Hugoniot data observed experimentally. The diminishing surfaces at 5.4 and $5.5 \mathrm{GPa}$ suggest the predominant formation of the NiAl intermetallic. ent lack of reaction signatures in similar spherical-shaped powders, these results suggest that the initiation of shockinduced reactions, or their inhibition, can be controlled through choice of extrinsic properties, such as the morphological configuration of reactants.

\section{ACKNOWLEDGMENTS}

The authors wish to acknowledge funding provided by an AFOSR/MURI Grant No. F49620-02-1-0382 and through a National Defense Science Engineering Graduate Fellowship awarded to Dan Eakins.

\section{APPENDIX: ESTIMATION OF REACTION EXTENT}

Consider a powder layer of total thickness $D$ subjected to a one-dimensional shock load, which has reacted incompletely, Fig. 6. The powder layer consists of a reacted zone, of thickness $d_{1}$, and unreacted zone, of thickness $d_{2}$. It is assumed that the behavior of the shock wave through the distributions of inert, and various reaction product phases is commutative, and can be equivalently represented by a series of parallel layers. Assuming also that the reacted zone denotes a region of increased shock velocity, but unaffected pressure (Ballotechnic model ${ }^{6,21}$ ), the shock velocities in the reacted and unreacted zone ( $U_{s}^{r x n}$ and $U_{s}^{o}$, respectively) will be distinct. The shock velocity observed experimentally will be given by

$$
U_{s}^{\mathrm{obs}}=\frac{D}{t},
$$

where $t$ is the shock wave travel time through the powder layer. Since the total transit time is the sum of the time through the reacted zone $t^{r x n}$ and unreacted zone $t^{o}$ such that

$$
\begin{aligned}
& t=t^{r x n}+t^{o}, \\
& t=\frac{d_{1}}{U_{s}^{r x n}}+\frac{d_{2}}{U_{s}^{o}},
\end{aligned}
$$

it follows:

$$
\begin{aligned}
& U_{s}^{\mathrm{obs}}=\frac{D}{\frac{d_{1}}{U_{s}^{r x n}}+\frac{d_{2}}{U_{s}^{o}}}, \\
& U_{s}^{\mathrm{obs}}=\frac{D\left(U_{s}^{r x n} U_{s}^{o}\right)}{d_{1} U_{s}^{o}+d_{2} U_{s}^{r x n}},
\end{aligned}
$$

or

$$
U_{s}^{\mathrm{obs}}=\frac{U_{s}^{r x n} U_{s}^{o}}{X^{r x n} U_{s}^{o}+\left(1-X^{r x n}\right) U_{s}^{r x n}},
$$

where the fraction of the total powder layer thickness that has undergone reaction $\left(d_{1} / D\right)$ is given by $X^{r x n}$. Solving for $X^{r x n}$ gives Eq. (1) in the main text.

It is also considered that the reacted zone contains multiple reaction product phases (Fig. 6), and that the shock velocity through the reacted zone $U_{s}^{r x n}$ is a combination of the shock velocities through those respective phases $\left(U_{s}^{\beta}\right.$, $U_{s}^{\delta}, U_{s}^{\gamma}$, etc.). This combination is performed in the same 
manner as shown above for Eq. (A1). For three phases, of thicknesses $d^{\beta}, d^{\delta}$, and $d^{\gamma}$, the shock transit time through the reacted region is

$$
\begin{aligned}
& t^{r x n}=t^{\beta}+t^{\delta}+t^{\gamma}, \\
& t^{r x n}=\frac{d^{\beta}}{U_{s}^{\beta}}+\frac{d^{\delta}}{U_{s}^{\delta}}+\frac{d^{\gamma}}{U_{s}^{\gamma}},
\end{aligned}
$$

where $t^{\beta}, t^{\delta}$, and $t^{\gamma}$ are the shock travel times through the phases $\beta, \delta$, and $\gamma$, respectively. The apparent shock velocity through the reacted zone is then given by

$$
\begin{aligned}
U_{s}^{r x n} & =\frac{d_{1}}{\frac{d^{\beta}}{U_{s}^{\beta}}+\frac{d^{\delta}}{U_{s}^{\delta}}+\frac{d^{\gamma}}{U_{s}^{\gamma}}}, \\
U_{s}^{r x n} & =\frac{U_{s}^{\beta} U_{s}^{\delta} U_{s}^{\gamma}}{X^{\beta} U_{s}^{\delta} U_{s}^{\gamma}+X^{\delta} U_{s}^{\beta} U_{s}^{\gamma}+X^{\gamma} U_{s}^{\beta} U_{s}^{\delta}},
\end{aligned}
$$

where $X^{\beta}, X^{\delta}$, and $X^{\gamma}$, are the respective fractions of the three phases relative to the reacted zone, i.e.,

$$
X^{\beta}+X^{\delta}+X^{\gamma}=1
$$

${ }^{1}$ S. S. Batsanov, G. S. Doronin, and S. V. Klochkov, Fiz. Goreniya Vzryva 22, 765 (1987).

${ }^{2}$ P. A. Taylor, M. B. Boslough, and Y. Horie, Modeling of Shock-Induced Chemistry in Nickel-Aluminum Systems (Elsevier Science, New York, 1987), pp. 395-398.

${ }^{3}$ M. B. Boslough, Chem. Phys. Lett. 160, 618 (1989).

${ }^{4}$ L. S. Bennett, F. Y. Sorrell, I. K. Simonsen, Y. Horie, and K. R. Iyer, Appl.
Phys. Lett. 61, 520 (1992).

${ }^{5}$ K. R. Iyer, L. S. Bennett, F. Y. Sorrell, and Y. Horie, Solid State Chemical Reactions at the Shock Front (American Institute of Physics, New York, 1994), pp. 1337-1340.

${ }^{6}$ R. A. Graham, M. U. Anderson, Y. Horie, S.-K. You, and G. T. Holman, Shock Waves 3, 79 (1993).

${ }^{7}$ S. S. Batsanov, M. F. Gogulya, and M. A. Brazhnikov, Fiz. Goreniya Vzryva 30, 107 (1994).

${ }^{8}$ S. S. Nabatov, G. E. Ivanchikhina, A. V. Kolesnikov, A. V. Kul'Bachevskii, and A. V. Lebedev, Chem. Phys. Rep. 14, 179 (1995).

${ }^{9}$ Y. Yang, R. D. Gould, Y. Horie, and K. R. Iyer, Appl. Phys. Lett. 70, 3365 (1997).

${ }^{10}$ N. N. Thadhani, R. A. Graham, T. Royal, E. Dunbar, M. U. Anderson, and G. T. Holman, J. Appl. Phys. 82, 1113 (1997).

${ }^{11}$ X. Xu and N. N. Thadhani, J. Appl. Phys. 96, 2000 (2004).

${ }^{12}$ K. S. Vandersall and N. N. Thadhani, J. Appl. Phys. 94, 1575 (2003).

${ }^{13}$ D. L. Gur'ev, Y. A. Gordopolov, and S. S. Batsanov, Combust., Explos. Shock Waves 42, 116 (2006).

${ }^{14}$ D. L. Gur'ev, Y. A. Gordopolov, S. S. Batsanov, A. G. Merzhanov, and V. E. Fortov, Appl. Phys. Lett. 88, 024102 (2006).

${ }^{15}$ W. H. Holt, J. W. Mock, M. U. Anderson, G. T. Holman, and R. A. Graham, AIP Conf. Proc. 370, 573 (1996).

${ }^{16}$ E. Dunbar, N. N. Thadhani, and R. A. Graham, J. Mater. Sci. 28, 2903 (1993).

${ }^{17}$ I. Song and N. N. Thadhani, Metall. Trans. A 23A, 41 (1992).

${ }^{18}$ D. E. Eakins and N. N. Thadhani, AIP Conf. Proc. 845, 1153 (2006).

${ }^{19}$ D. E. Eakins and N. N. Thadhani, Mater. Res. Soc. Symp. Proc. 896, 191 (2006).

${ }^{20}$ R. G. McQueen, S. P. Marsh, J. W. Taylor, J. N. Fritz, and W. J. Carter, The Equation of State of Solids from Shock Wave Studies, High Velocity Impact Phenomena (Academic, New York, 1970).

${ }^{21}$ L. S. Bennett and Y. Horie, Shock Waves 4, 127 (1994).

${ }^{22}$ PlotData, v. 2.0, Sandia National Laboratories.

${ }^{23}$ M. Yoshida and N. N. Thadhani, Study of Shock Induced Solid State Reactions by Recovery Experiments and Measurements of Hugoniot and Sound Velocity (Elsevier Science, Amsterdam, 1991), pp. 586-592. 